\title{
1 Hybrid Organic/Inorganic Perovskite-Polymer Nanocomposites: 2 Toward the Enhancement of Structural and Electrical Properties
}

\author{
3 Alberto Privitera, ${ }^{*},{ }^{\dagger}$ Marcello Righetto, ${ }^{\dagger}$ Michele De Bastiani, ${ }^{\dagger}$ Francesco Carraro, ${ }^{\dagger}{ }^{\circ}$ Marzio Rancan, ${ }^{\dagger, \S}$ \\ ${ }_{4}$ Lidia Armelao, ${ }^{\dagger, \S}$ Gaetano Granozzi, ${ }^{\dagger}{ }^{\dagger}$ Renato Bozio, ${ }^{\dagger}$ and Lorenzo Franco ${ }^{\dagger \odot}$ \\ $5{ }^{\dagger}$ Department of Chemical Science and U.R. INSTM, University of Padova, Via Marzolo 1, I-35131 Padova, Italy \\ $6{ }^{\ddagger}$ King Abdullah University of Science and Technology (KAUST), KAUST Solar Center (KSC), Division of Physical Sciences and \\ 7 Engineering (PSE), Thuwal 23955-6900, Kingdom of Saudi Arabia \\ $8{ }^{\S}$ ICMATE-CNR and U.R. INSTM, University of Padova, Via Marzolo 1, I-35131 Padova, Italy
}

10 ABSTRACT: Hybrid organic/inorganic perovskite nanoparticles (NPs) have garnered remarkable research attention because of their promising photophysical properties. New and interesting properties emerge after combining perovskite NPs with semiconducting materials. Here, we report the synthesis and investigation of a composite material obtained by mixing $\mathrm{CH}_{3} \mathrm{NH}_{3} \mathrm{PbBr}_{3}$ nanocrystals with the semiconducting polymer poly(3hexylthiophene) (P3HT). By the combination of structural techniques and optical and magnetic spectroscopies we observed multiple effects of the perovskite NPs on the P3HT: (i) an enlargement of P3HT crystalline domains, (ii) a strong p-doping of the P3HT, and (iii) an enhancement of interchain order typical of $\mathrm{H}$-aggregates. These observations open a new avenue toward innovative perovskite NP-based applications.

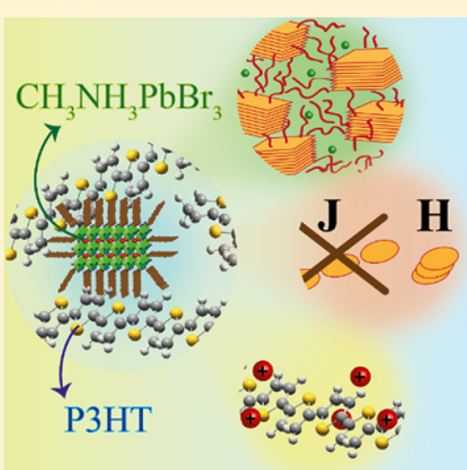

${ }_{21}^{20} \mathrm{~V}$ ery recently there has been a huge growth of interest in hybrid perovskite materials. ${ }^{1-8}$ In analogy with confine22 ment effects in conventional semiconductors, exciting new 23 physical properties emerge with the miniaturization of hybrid 24 perovskites materials to the nanometer scale. ${ }^{9-16}$ Among these 25 properties, higher oscillator strength, size tunability of energy 26 levels, Auger-assisted charge transfer, and phonon bottleneck 27 multiexciton generation represent only a few of the many 28 features which provide perovskite nanostructures an appealing 29 interest for optoelectronic applications. ${ }^{17-22}$ Moreover, as 30 discovered in conventional semiconductors, new properties 31 can be triggered by coupling inorganic nanoparticles (NPs) 32 with other semiconducting materials. ${ }^{23}$ Among the many 33 nanostructured composites, those including NPs and conduct34 ing polymers have played a pivotal role in the last two 35 decades. ${ }^{23-25}$ For example, in organic solar cells, blending NPs 36 with conducting polymers has contributed toward the enhance37 ment of light absorption, fast exciton diffusion and dissociation, 38 as well as balanced electron-hole transport. ${ }^{26-29}$ A similar 39 success has been obtained also in thermoelectric applications, 40 where NP incorporation improves device performances. ${ }^{30}$ In 41 Li-ion batteries, NP/polymer nanocomposites used at the 42 cathode or anode have contributed to a significant improve43 ment of capacity and cyclability of the devices. ${ }^{31}$ Finally, NP 44 incorporation has facilitated high luminous yield, color 45 tunability, and low operation voltage for organic light-emitting 46 diodes. $^{32}$
Despite the promising response of inorganic NP/conducting 47 polymer nanocomposites in optoelectronic devices, only few 48 examples have so far examined the benefits given by hybrid 49 perovskite nanostructures to the crystallinity, morphology, and 50 photophysics of polymers. ${ }^{33}$ In particular, the control over 51 crystallinity in partially disordered materials is of paramount 52 importance for achieving highly performing optoelectronic 53 devices. $^{34,35}$ Indeed, the formation of larger ordered polymer 54 domains has a strong impact on photophysical processes, such 55 as exciton and polaron dynamics. ${ }^{36-39}$

Within this scenario, we investigated a nanocomposite 57 material of methylammonium lead bromide $\left(\mathrm{CH}_{3} \mathrm{NH}_{3} \mathrm{PbBr}_{3}\right) 58$ perovskite nanoparticles (PNPs) blended with poly(3-hexylth- 59 iophene) (P3HT) (hereafter P3HT/PNPs). We explored the 60 effect of the PNPs on the morphology and the photophysics of 61 P3HT by combining optical and magnetic spectroscopies with 62 $\mathrm{X}$-ray diffraction, electron microscopy/spectroscopy, and 63 elemental analysis. Our study disclosed a triple influence of 64 the PNPs on the P3HT properties. First, the P3HT crystalline 65 domains notably increased in dimensions when blended with 66 the PNPs with respect to the pristine conditions. Second, a 67 strong p-doping of the P3HT is induced by the PNPs. Third, a 68 large area of $\pi$-stacking between adjacent monomers of P3HT, 69 forming $\mathrm{H}$-aggregates, is generated by the presence of the 70

Received: November 20, 2017

Accepted: November 30, 2017

Published: November 30, 2017 

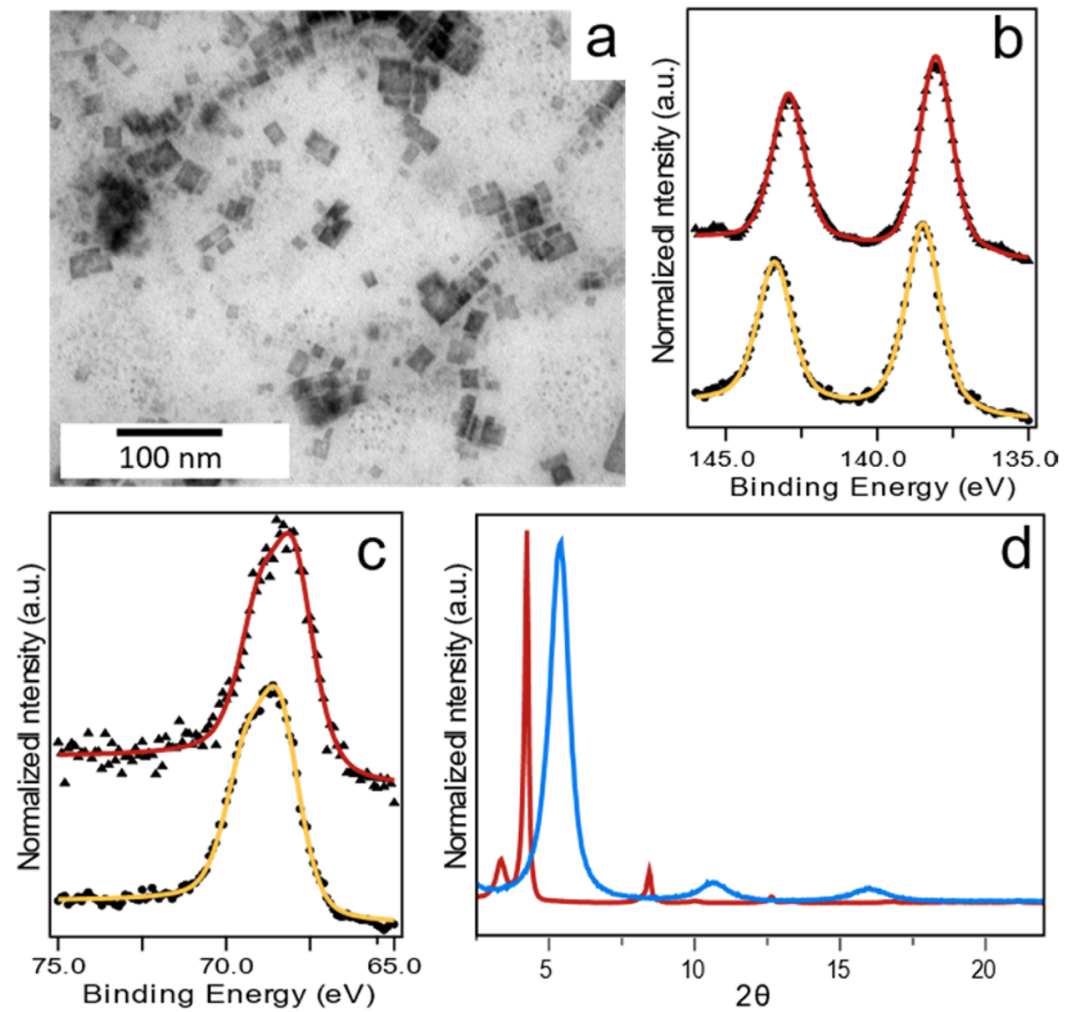

Figure 1. (a) TEM image of a thin film of the P3HT/PNPs blend. (b, c) Pb $4 \mathrm{f}$ and $\mathrm{Br} 3 \mathrm{~d}$ photoemission raw spectra and total fit of PNPs (black circles, orange line) and P3HT/PNPs (black triangles, red line) samples, respectively. (d) PXRD spectra of pristine P3HT film (blue line) and P3HT/PNPs blend (red line).

71 PNPs. The combination of these effects might open the way to 72 novel functionalities of the composite material and to the 73 development of highly efficient optoelectronic devices.

74 We synthesized PNPs following the well-established ligand75 assisted reprecipitation (LARP) technique. ${ }^{20}$ The suspended $76 \mathrm{NP}$ solution was centrifuged to discard the precipitates, and a 77 bright blue-green photoluminescence, fingerprint of PNPs, was 78 observed in the supernatant solution (more details on the 79 synthesis are reported in the Supporting Information). The as80 synthesized PNPs show an absorption edge at $510 \mathrm{~nm}$ with an 81 intense photoluminescence peak at $520 \mathrm{~nm}$, blue-shifted with 82 respect to bulk $\mathrm{CH}_{3} \mathrm{NH}_{3} \mathrm{PbBr}_{3}$ and in agreement with the 83 quantum confinement effect (see Figure SI_1a). Powder XRD 84 (PXRD) of PNPs matches well the cubic phase $(P m \overline{3} m)$ of ${ }_{85} \mathrm{CH}_{3} \mathrm{NH}_{3} \mathrm{PbBr}_{3}$ (see Figure SI_1b). ${ }^{20}$

86 With the aim of investigating the effects induced by the PNPs 87 on the morphology and the optoelectronic properties of an 88 embedding conducting polymer, we prepared a composite 89 material P3HT/PNPs by mixing PNPs and P3HT (details in 90 the Supporting Information).

91 TEM images of the P3HT/PNPs composite clearly exhibit a 92 uniform dispersion of the PNPs in the polymer matrix (Figures 93 la and SI_2b), indicating a homogeneous mixing in the 94 composite material. In addition, the presence of perovskite 95 nanoplatelets of average side of $10-30 \mathrm{~nm}$ is observed. Their 96 presence is also confirmed by XRD and optical analysis (see 97 below). Additional evidence of the uniform PNPs dispersion is 98 provided by SEM micrographs (Figure SI_3) and energy 99 dispersive X-ray spectroscopy (EDS, Figure SI_4) which 100 confirms a homogeneous distribution of $\mathrm{Br}$ and $\overline{\mathrm{Pb}}$ with a 101 matching ratio corresponding to hybrid perovskites $(\mathrm{Br} / \mathrm{Pb}=$ $1023.5)$.
We also determined the chemical composition of pristine 103 PNPs and the P3HT/PNPs composite by X-ray photoemission 104 spectroscopy (XPS). Surface elemental composition (atom \%) 105 determined by XPS quantitative analysis confirmed the 106 presence of the PNPs with a calculated ratio $\mathrm{Br} / \mathrm{Pb}$ of 2.5107 and 3.3 for the PNPs and P3HT/PNPs, respectively, in good 108 agreement with EDS results. The small difference in the 109 calculated surface $\mathrm{Br} / \mathrm{Pb}$ ratio can be attributed to the presence 110 of P3HT and to surface effects in the composite material. 111 Pristine PNPs showed two symmetric peaks attributed to $\mathrm{Pb} 112$ $4 \mathrm{f} 7 / 2$ and $\mathrm{Pb} 4 \mathrm{f5} / 2$ level at binding energies (BE) of 138.5 and 113 $143.4 \mathrm{eV}$, respectively (Figures $1 \mathrm{~b}$ and SI_5). The deconvo- 114 lution of the $\mathrm{Pb} 4 \mathrm{f}$ confirms that no undesired $\mathrm{Pb}$ oxides phases 115 were formed. ${ }^{40}$ Interestingly, we observe a shift of $0.4 \mathrm{eV}$ to 116 lower BE of the perovskite's photoemission lines (Figures 1c 117 and SI_6). This shift can be induced by the presence of an 118 electronic interaction between the PNPs and the P3HT which 119 generates an excess of negative charges on the PNPs, thus 120 lowering the binding energy.

To further characterize the effect of the interaction between 122 PNPs and the polymer, we recorded the PXRD patterns of 123 $\mathrm{P} 3 \mathrm{HT} / \mathrm{PNPs}$ and pristine P3HT. The pristine P3HT shows an 124 intense reflection at $5.4^{\circ}(100)$ and two smaller ones at $10.6^{\circ} 125$ (200) and $16.0^{\circ}$ (300) commonly attributed to the lamellar 126 ordering of the polymer chains induced by the $\pi$-stacking of the 127 aromatic rings (Figure 1d). The P3HT/PNPs composite shows 128 a clearly different diffraction pattern with respect to the pristine 129 polymer. In particular, a severe narrowing of the XRD 130 reflections and a shift to lower angles are observed. The 131 narrowing of the peaks is attributed to a structural rearrange- 132 ment of the polymer with the formation of larger crystalline 133 domains. Concerning the shift of the peaks, some possible 134 
135 explanations are reported in the literature. Doping of P3HT (p136 doping) has been reported to produce a shift of the low-angle 137 reflections with respect to the pristine condition, ${ }^{41,42}$ and it was 138 also demonstrated that when the doping overcomes a critical 139 threshold, P3HT can undergo a deep structural rearrange140 ment. $^{43}$ These explanations do not seem to be fully applicable 141 to our case. However, for our purposes, the most important 142 result from XRD is the detection of an enhanced P3HT 143 crystallinity in the blend.

144 Finally, by magnifying the region between $10^{\circ}$ and $50^{\circ}$ 145 (Figure SI_7), a series of very low-intensity peaks is visible. 146 These low-intensity peaks are associated with the PNPs. Similar 147 features for PNPs were observed in the presence of perovskite 148 nanoplatelets. ${ }^{44}$

149 To further investigate the increased crystallinity of the P3HT 150 in the blend with PNPs, we applied electron paramagnetic 151 resonance (EPR) spectroscopy, which can provide structural 152 and dynamical information about charge carriers (polarons) in 153 P3HT blends. ${ }^{45}$

154 The EPR spectra of the neat P3HT film and the P3HT/
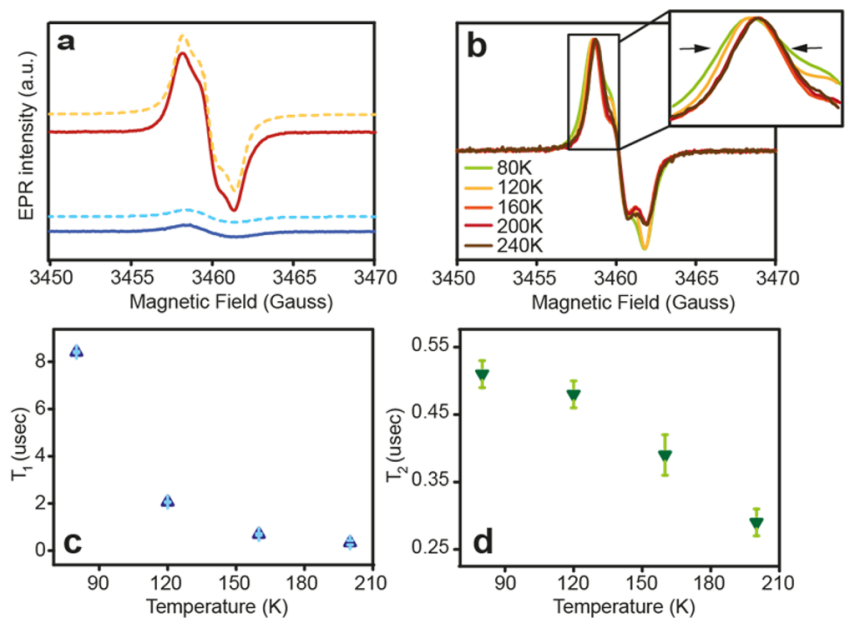

Figure 2. (a) EPR spectra of the neat P3HT film (blue line) and $\mathrm{P} 3 \mathrm{HT} / \mathrm{PNPs}$ blend (red line) recorded at $80 \mathrm{~K}$ and best fit spectral simulation (light blue and orange dotted lines). (b) Normalized EPR spectra of P3HT/PNPs blend recorded at various temperatures. The inset illustrates the narrowing of the EPR line at increasing temperatures. (c, d) $T_{1}$ and $T_{2}$ electron spin relaxation times as a function of temperature for P3HT/PNPs blend.

156 neat P3HT film (blue line), only a weak EPR peak is detected. 157 From the spectral simulation the g-tensor typical of spin $1 / 2$ 158 positive charge carriers (polarons) in $\mathrm{P} 3 \mathrm{HT}$ is obtained ( $\mathrm{g}$ 159 tensor principal values: 2.001, 2.002, and 2.003). ${ }^{46}$ The 160 presence of this small signal is attributed to a partial intrinsic 161 oxidation of the polymer in air. ${ }^{47}$ In contrast, the P3HT/PNPs 162 blend shows a strong EPR line with the same g-tensor 163 indicating a much stronger oxidation, i.e., a p-doping, of the 164 polymer. ${ }^{46}$ The p-doping of P3HT in the blend is consistent 165 with the observed XPS shift previously discussed. We explain 166 the excess of positive polarons on the P3HT as the result of 167 either a thermal or an ambient light-induced electron transfer 168 from the P3HT to the perovskite NPs. The presence of an 169 electronic interaction between the two components of the 170 blend is demonstrated also by PL quenching measurements 171 (Figure SI_8). We observed a complete quenching of the PL of 172 both the P3HT and the PNPs when they are mixed together in the blend. From the EPR results, the PL quenching can be 173 attributed to the electron transfer from the P3HT to the PNPs. 174 A multitude of different causes that promote this electron 175 transfer can be envisaged. A relevant role can be represented by 176 trap states induced by surface defects in PNPs, which are 177 reported to affect the photophysical properties of the NPs. ${ }^{48,49} 178$ These trap states may act as interfacial sink for the transferred 179 electrons, providing an excess of positive charges left on the 180 P3HT. Additionally, the generated polarons are stabilized by 181 the high dielectric permittivity of the perovskite that hinders 182 the back electron-transfer process. As well as the increased 183 number of polarons, a secondary effect induced by the presence 184 of PNPs is represented by an anomalous narrowing of EPR line 185 width. We rationalize this evidence as being due to a motional 186 narrowing regime of the polarons in the blend induced by a 187 higher mobility with respect to the neat P3HT film. ${ }^{50} 188$

We confirm such an explanation by investigating in more 189 detail the dynamics of the polarons in the blend recording EPR 190 spectra in a broad range of temperatures, from 80 to $240 \mathrm{~K} 191$ (Figure 2b). When the temperature is increased from $80 \mathrm{~K}$, a 192 contraction of the EPR line width is observed, while the g- 193 tensor anisotropy is substantially unvaried. This observation is 194 likely due to a thermally activated charge carriers diffusion 195 along and between polymer chains. ${ }^{50}$ The higher charge 196 diffusion rate induces a more efficient averaging of broadening 197 due to hyperfine coupling and an EPR line width narrowing. ${ }^{58} 198$ However, if the motion of the polarons occurs within equally 199 oriented molecules in a crystalline domain, it does not result in 200 an averaging of the g-anisotropy. Therefore, in the presence of a 201 high crystallinity of the sample, which allows a high charge 202 mobility, the resulting EPR spectrum of the polarons shows the 203 full g-tensor anisotropy with a narrow line width. Electron spin 204 relaxation time measurements provide further confirmation of 205 the temperature-dependent enhanced mobility of polarons in 206 the P3HT/PNPs blend. Figure 2c,d shows a decrease of 207 electron spin-lattice and spin-spin relaxation times as a 208 function of temperature. In the P3HT/PNPs blend, polaron 209 electron spin relaxation is governed by spin dynamics. Indeed, 210 in organic systems, electron spin relaxation is caused by local 211 magnetic field fluctuations mainly due to magnetic dipole- 212 dipole interactions. ${ }^{50}$ A faster charge carriers' diffusion along 213 and between polymer chains increases the rate of the local 214 magnetic field fluctuations experienced by the polaron unpaired 215 electron. A higher rate of these fluctuations is the cause of the 216 decrease of the polaron spin relaxation times. Therefore, at 217 increasing temperatures, a faster polaron diffusion lowers both 218 electron spin-lattice and spin-spin relaxation times. 219

The polaron diffusion along and between P3HT chains is 220 strongly influenced by the packing of the polymer chains within 221 the crystalline domains, which in turn can be determined by the 222 relative orientation between the $\mathrm{P} 3 \mathrm{HT}$ chains. We used 223 ultraviolet-visible (UV-vis) spectroscopy to investigate the 224 degree of interchain order and the relative orientation of 225 transition dipoles associated with P3HT units. This analysis 226 takes advantage of the coupled Frenkel excitons model 227 developed by Spano for semiconducting polymers. ${ }^{51}$ In 228 particular, models involving $\mathrm{H}$ - and J-aggregates were proposed 229 to predict optical properties of P3HT films. ${ }^{51,52}$ Upon 230 deposition, interactions between monomers can be either 231 interchain or intrachain. Hence, the competition between 232 through-bond and through-space excitonic coupling contains 233 information on the aggregation of P3HT. In particular, a 234 dominant interchain coupling, associated with a high degree of 235 
236 interchain order, would result in cofacial $\mathrm{H}$-aggregate signatures 237 in the optical spectrum. On the other hand, a dominant 238 intrachain coupling, reflecting higher intrachain order, would 239 result in J-aggregate signatures. Spano et al. demonstrated that 240 electronic dispersion of molecular levels, associated with 241 aggregate formation, results in vibronic bands. ${ }^{51,52}$ The relative 242 intensities of vibronic bands are a sensitive probe for polymer 243 inner structure. Indeed, the ratio of the first two vibronic peak 244 intensities in the absorption spectrum $\left(I_{00} / I_{01}\right)$ is correlated 245 with the degree of inter/intrachain order, i.e., the lower this 246 ratio, the higher the interchain order. UV-vis spectra recorded 247 for pristine P3HT and P3HT/PNP film are shown in Figure 3a.
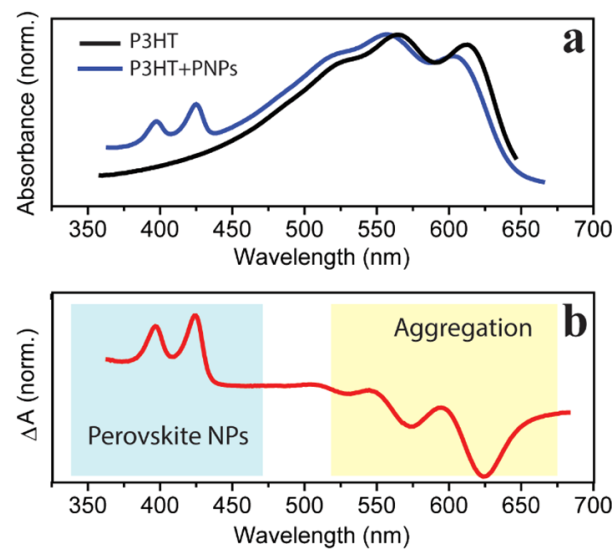

Figure 3. (a) UV-vis absorption spectra of P3HT (black) and P3HT/ PPs blend (blue). (b) Difference between UV-vis spectra of P3HT with and without perovskite NPs. Changes in the vibronic structure of P3HT account for different aggregation, while appearance of novel absorption peaks on the blue absorption tail are ascribed to perovskite nanoplatelets.

248 In Figure 3b, we present the difference between the spectrum 249 of P3HT with and without PNPs to magnify the differences. 250 This subtraction reveals a redistribution of relative intensities of 251 vibronic bands, associated with a microscopic ordering of the 252 aggregated polymer. The strong decrease of $I_{00}$ suggests an 253 augmented interchain order, confirming EPR and XRD data. 254 According to refs 51 and 52, we can quantitatively estimate the 255 exciton bandwidth associated with $\mathrm{H}$-aggregates formation 256 using relation 1 :

257

$$
\frac{I_{00}}{I_{01}} \approx \frac{n_{01}}{n_{00}}\left(\frac{1-0.24 W / E_{\mathrm{p}}}{1+0.073 W / E_{\mathrm{p}}}\right)^{2}
$$

258 where $W$ is the exciton bandwidth and $E_{\mathrm{p}}$ is the frequency of 259 the coupled normal mode; $n$ are the refractive indexes. By using 260 parameters similar to those in ref 51 , we estimate a 3 -fold 261 increment in the exciton bandwidth, from 24 to $67 \mathrm{meV}$, when 262 PNPs are blended with P3HT. This result indicates that the 263 presence of PNPs directs crystallization toward the formation 264 of $\mathrm{H}$-aggregates.

265 Moreover, two additional peaks observed at shorter wave266 length are assigned to perovskite nanoplatelets. ${ }^{44}$ We suggest 267 that these features originate from the exfoliation of PNPs 268 during the deposition process which results in the formation of 269 mono- and bilayered perovskite nanoplatelets in agreement 270 with the layered nature of perovskite materials. ${ }^{44}$

271 Clearly, together with standard parameters (such as the 272 choice of solvent and of film deposition conditions ${ }^{23}$ ), the interfacial regions between the PNPs and the P3HT rule the 273 crystallization process of the polymer and actively modify the 274 P3HT aggregation. The combination of the good miscibility 275 between the two components of the blend, the nanometric size 276 of PNPs, and the presence of perovskite nanoplatelets increases 277 the interfacial regions and bolsters the formation of large 278 crystalline domains of $\mathrm{H}$-aggregates.

279

To conclude, in this work we presented an in-depth study of 280 a polymer/nanoparticle composite material, based on semi- 281 conducting polymer P3HT and methylammonium lead bro- 282 mide perovskite nanoparticles. After having demonstrated the 283 good miscibility between the P3HT and PNPs and the optimal 284 homogeneity of the composite by using TEM, SEM, EDS, and 285 XPS analysis, we investigated how the morphological and 286 electrical properties of the P3HT are enhanced by the presence 287 of PNPs. The combined use of X-ray diffraction, EPR 288 spectroscopy, and optical analysis revealed a 3-fold contribution 289 of the PNPs on the properties of P3HT: (i) an increment of the 290 P3HT crystalline domains, (ii) a p-doping of the P3HT, and 291 (iii) the extended formation of $\pi$-stacking between adjacent 292 monomers of P3HT (H-aggregates). Our results may be 293 potentially extended to other semiconducting polymers 294 blended with hybrid organic perovskite NPs. Further 295 experimental work is currently underway to test this hypothesis. 296 In addition, because the structural and electronic control of 297 conducting polymers is of paramount importance for achieving 298 high-performance optoelectronic devices, our composite ma- 299 terial is promising for the design of new highly performing 300 devices.

\section{ASSOCIATED CONTENT}

\section{S Supporting Information}

303

The Supporting Information is available free of charge on the 304 ACS Publications website at DOI: 10.1021/acs.jpclett.7b03077. 305

Details of experimental methods, characterization of 306 PNPs, EDS elemental maps, XPS spectra, and PXRD 307 patterns of the studied samples (PDF)

\section{AUTHOR INFORMATION}

\section{Corresponding Author}

*E-mail: alberto.privitera@phd.unipd.it.

ORCID $\odot$

Alberto Privitera: 0000-0002-7062-8077

Marcello Righetto: 0000-0001-5507-1445

Francesco Carraro: 0000-0001-8485-4676

Gaetano Granozzi: 0000-0002-9509-6142

Renato Bozio: 0000-0001-7274-9190

Lorenzo Franco: 0000-0003-3548-4423

Notes

The authors declare no competing financial interest.

\section{ACKNOWLEDGMENTS}

Financial support from Centro Studi di Economia e Tecnica 322 dell'Energia Giorgio Levi Cases of the University of Padova is 323 gratefully acknowledged (project OPERA). G.G. and F.C. 324 thank the MAECI (Ministero degli Affari Esteri e della 325 Cooperazione Internazionale) for the support through the 326 bilateral Italy-China GRAPE-MAT project. Finally, we thank 327 Prof. Osman Bakr for fruitful discussion and suggestions on our 328 results. 
331 (1) Brenner, T. M.; Egger, D. A.; Kronik, L.; Hodes, G.; Cahen, D. 332 Hybrid Organic-Inorganic Perovskites: Low-Cost Semiconductors 333 with Intriguing Charge-Transport Properties. Nat. Rev. Mater. 2016, $3341,15007$.

335 (2) Zhao, Y.; Zhu, K. Organic-Inorganic Hybrid Lead Halide 336 Perovskites for Optoelectronic and Electronic Applications. Chem. Soc. 337 Rev. 2016, 45, 655-689.

338 (3) Shi, Z.; Guo, J.; Chen, Y.; Li, Q.; Pan, Y.; Zhang, H.; Xia, Y.; 339 Huang, W. Lead-Free Organic-Inorganic Hybrid Perovskites for 340 Photovoltaic Applications: Recent Advances and Perspectives. Adv. 341 Mater. 2017, 29, 1605005.

342 (4) Chen, Q.; De Marco, N.; Yang, Y.; Song, T.-B.; Chen, C.-C.; 343 Zhao, H.; Hong, Z.; Zhou, H.; Yang, Y. Under the Spotlight: The 344 Organic-Inorganic Hybrid Halide Perovskite for Optoelectronic 345 Applications. Nano Today 2015, 10, 355-396.

346 (5) Ye, M.; He, C.; Iocozzia, J.; Liu, X.; Cui, X.; Meng, X.; Rager, M.; 347 Hong, X.; Liu, X.; Lin, Z. Recent Advances in Interfacial Engineering 348 of Perovskite Solar Cells. J. Phys. D: Appl. Phys. 2017, 50, 373002.

349 (6) He, M.; Zheng, D.; Wang, M.; Lin, C.; Lin, Z. High Efficiency 350 Perovskite Solar Cells: from Complex Nanostructure to Planar 351 Heterojunction. J. Mater. Chem. A 2014, 2, 5994-6003.

352 (7) He, M.; Li, B.; Cui, X.; Jiang, B.; He, Y.; Chen, Y.; O’Neil, D.; 353 Szymanski, P.; Ei-Sayed, M. A.; Huang, J.; et al. Meniscus-Assisted 354 Solution Printing of Large-Grained Perovskite Films for High355 Efficiency Solar Cells. Nat. Commun. 2017, 8, 16045.

356 (8) He, M.; Pang, X.; Liu, X.; Jiang, B.; He, Y.; Snaith, H.; Lin, Z. 357 Monodisperse Dual-Functional Upconversion Nanoparticles Enabled 358 Near-Infrared Organolead Halide Perovskite Solar Cells. Angew. Chem., 359 Int. Ed. 2016, 55, 4280-4284.

360 (9) González-Carrero, S.; Galian, R. E.; Pérez-Prieto, J. Organometal 361 Halide Perovskites: Bulk Low-Dimension Materials and Nanoparticles. 362 Part. Part. Syst. Charact. 2015, 32, 709-720.

363 (10) Gonzalez-Carrero, S.; Galian, R. E.; Pérez-Prieto, J. Organic364 Inorganic and All-Inorganic Lead Halide Nanoparticles [Invited]. Opt. 365 Express 2016, 24, A285-A301.

366 (11) Luo, B.; Pu, Y.-C.; Yang, Y.; Lindley, S. A.; Abdelmageed, G.; 367 Ashry, H.; Li, Y.; Li, X.; Zhang, J. Z. Synthesis, Optical Properties, and 368 Exciton Dynamics of Organolead Bromide Perovskite Nanocrystals. J. 369 Phys. Chem. C 2015, 119, 26672-26682.

370 (12) Zheng, K.; Ž́́dek, K.; Abdellah, M.; Messing, M. E.; Al-Marri, M. 371 J.; Pullerits, T. Trap States and Their Dynamics in Organometal 372 Halide Perovskite Nanoparticles and Bulk Crystals. J. Phys. Chem. C 373 2016, 120, 3077-3084.

374 (13) Schmidt, L. C.; Pertegás, A.; González-Carrero, S.; 375 Malinkiewicz, O.; Agouram, S.; Mínguez Espallargas, G.; Bolink, H. 376 J.; Galian, R. E.; Pérez-Prieto, J. Nontemplate Synthesis of $377 \mathrm{CH}_{3} \mathrm{NH}_{3} \mathrm{PbBr}_{3}$ Perovskite Nanoparticles. J. Am. Chem. Soc. 2014, 378 136, 850-853.

379 (14) Mittal, M.; Jana, A.; Sarkar, S.; Mahadevan, P.; Sapra, S. Size of 380 the Organic Cation Tunes the Band Gap of Colloidal Organolead 381 Bromide Perovskite Nanocrystals. J. Phys. Chem. Lett. 2016, 7, 32703823277.

383 (15) Privitera, A.; Righetto, M.; Bozio, R.; Franco, L. The Central 384 Role of Ligands in Electron Transfer from Perovskite Nanocrystals. 385 MRS Advances 2017, 2, 2327.

386 (16) Lamberti, F.; Litti, L.; De Bastiani, M.; Sorrentino, R.; Gandini, 387 M.; Meneghetti, M.; Petrozza, A. High-Quality, Ligands-Free, Mixed388 Halide Perovskite Nanocrystals Inks for Optoelectronic Applications. 389 Adv. Energy Mater. 2017, 7, 1601703.

390 (17) Huang, H.; Susha, A. S.; Kershaw, S. V.; Hung, T. F.; Rogach, A. 391 L. Control of Emission Color of High Quantum Yield $\mathrm{CH}_{3} \mathrm{NH}_{3} \mathrm{PbBr}_{3}$ 392 Perovskite Quantum Dots by Precipitation Temperature. Adv. Sci. 393 2015, 2, 1500194.

394 (18) Martinez-Sarti, L.; Koh, T. M.; La-Placa, M.-G.; Boix, P. P.; 395 Sessolo, M.; Mhaisalkar, S. G.; Bolink, H. J. Efficient Photoluminescent 396 Thin Films Consisting of Anchored Hybrid Perovskite Nanoparticles. 397 Chem. Commun. 2016, 52, 11351-11354.
(19) Sichert, J. A.; Tong, Y.; Mutz, N.; Vollmer, M.; Fischer, S.; 398 Milowska, K. Z.; García Cortadella, R.; Nickel, B.; Cardenas-Daw, C.; 399 Stolarczyk, J. K.; et al. Quantum Size Effect in Organometal Halide 400 Perovskite Nanoplatelets. Nano Lett. 2015, 15, 6521-6527. 401

(20) Zhang, F.; Zhong, H.; Chen, C.; Wu, X.-g.; Hu, X.; Huang, H.; 402 Han, J.; Zou, B.; Dong, Y. Brightly Luminescent and Color-Tunable 403 Colloidal $\mathrm{CH}_{3} \mathrm{NH}_{3} \mathrm{PbX}_{3}(\mathrm{X}=\mathrm{Br}, \mathrm{I}, \mathrm{Cl})$ Quantum Dots: Potential 404 Alternatives for Display Technology. ACS Nano 2015, 9, 4533-4542. 405 (21) Veldhuis, S. A.; Boix, P. P.; Yantara, N.; Li, M.; Sum, T. C.; 406 Mathews, N.; Mhaisalkar, S. G. Perovskite Materials for Light-Emitting 407 Diodes and Lasers. Adv. Mater. 2016, 28, 6804-6834.

(22) Chen, Y.; Yang, D.; Yoon, Y. J.; Pang, X.; Wang, Z.; Jung, J.; He, 409 Y.; Harn, Y. W.; He, M.; Zhang, S.; et al. Hairy Uniform Permanently 410 Ligated Hollow Nanoparticles with Precise Dimension Control and 411 Tunable Optical Properties. J. Am. Chem. Soc. 2017, 139, 12956- 412 12967.

(23) Saini, P. Fundamentals of Conjugated Polymer Blends, Copolymers 414 and Composites: Synthesis, Properties, and Applications; Wiley: Beverly, 415 MA, 2015 .

(24) Eftekhari, A. Nanostructured Conductive Polymers; Wiley: 417 Chichester, U.K., 2011.

(25) Pan, S.; He, L.; Peng, J.; Qiu, F.; Lin, Z. Chemical-Bonding- 419 Directed Hierarchical Assembly of Nanoribbon-Shaped Nanocompo- 420 sites of Gold Nanorods and Poly(3-hexylthiophene). Angew. Chem., 421 Int. Ed. 2016, 55, 8686-8690.

(26) Carey, G. H.; Abdelhady, A. L.; Ning, Z.; Thon, S. M.; Bakr, O. 423 M.; Sargent, E. H. Colloidal Quantum Dot Solar Cells. Chem. Rev. 424 2015, 115, 12732-12763.

(27) Kamat, P. V. Quantum Dot Solar Cells. Semiconductor 426 Nanocrystals as Light Harvesters. J. Phys. Chem. C 2008, 112, 427 18737-18753.

(28) Kamat, P. V. Quantum Dot Solar Cells. The Next Big Thing in 429 Photovoltaics. J. Phys. Chem. Lett. 2013, 4, 908-918. 430

(29) Sargent, E. H. Colloidal Quantum Dot Solar Cells. Nat. 431 Photonics 2012, 6, 133-135.

(30) McGrail, B. T.; Sehirlioglu, A.; Pentzer, E. Polymer Composites 433 for Thermoelectric Applications. Angew. Chem., Int. Ed. 2015, 54, 434 $1710-1723$.

(31) Sengodu, P.; Deshmukh, A. D. Conducting Polymers and Their 436 Inorganic Composites for Advanced Li-Ion Batteries: A Review. RSC 437 Adv. 2015, 5, 42109-42130.

(32) Buckley, A. Organic Light-emitting Diodes (OLEDs): Materials, 439 Devices and Applications; Elsevier Science \& Technology: Cambridge, 440 U.K., 2013.

(33) Masi, S.; Colella, S.; Listorti, A.; Roiati, V.; Liscio, A.; Palermo, 442 V.; Rizzo, A.; Gigli, G. Growing Perovskite into Polymers for Easy- 443 Processable Optoelectronic Devices. Sci. Rep. 2015, 5, 7725.

(34) Bansal, N.; Reynolds, L. X.; MacLachlan, A.; Lutz, T.; Ashraf, R. 445 S.; Zhang, W.; Nielsen, C. B.; McCulloch, I.; Rebois, D. G.; Kirchartz, 446 T.; et al. Influence of Cystallinity and Energetics on Charge Separation 447 in Polymer-Inorganic Nanocomposite Films for Solar Cells. Sci. Rep. 448 2013, 3, 1531.

(35) Kumar, V.; Kalia, S.; Swart, H. C. Conducting Polymer Hybrids; 450 Springer International Publishing: Cham, Switzerland, 2016.

(36) Liu, Y.; Zhao, J.; Li, Z.; Mu, C.; Ma, W.; Hu, H.; Jiang, K.; Lin, 452 H.; Ade, H.; Yan, H. Aggregation and Morphology Control Enables 453 Multiple Cases of High-Efficiency Polymer Solar Cells. Nat. Commun. 454 2014, 5, 5293.

(37) Chang, J.-F.; Sun, B.; Breiby, D. W.; Nielsen, M. M.; Sölling, T. 456 I.; Giles, M.; McCulloch, I.; Sirringhaus, H. Enhanced Mobility of 457 Poly(3-hexylthiophene) Transistors by Spin-Coating from High- 458 Boiling-Point Solvents. Chem. Mater. 2004, 16, 4772-4776. 459

(38) Sirringhaus, H.; Brown, P. J.; Friend, R. H.; Nielsen, M. M.; 460 Bechgaard, K.; Langeveld-Voss, B. M. W.; Spiering, A. J. H.; Janssen, R. 461 A. J.; Meijer, E. W.; Herwig, P.; et al. Two-Dimensional Charge 462 Transport in Self-Organized, High-Mobility Conjugated Polymers. 463 Nature 1999, 401, 685-688.

(39) Silva, C.; Russell, D. M.; Dhoot, A. S.; Herz, L. M.; Daniel, C.; 465 Greenham, N. C.; Arias, A. C.; Setayesh, S.; Mullen, K.; Friend, R. H. 466 
467 Exciton and Polaron Dynamics in a Step-Ladder Polymeric Semi468 conductor: The Influence of Interchain Order. J. Phys.: Condens. Matter 469 2002, 14, 9803.

470 (40) Lindblad, R.; Bi, D.; Park, B.-w.; Oscarsson, J.; Gorgoi, M.; 471 Siegbahn, H.; Odelius, M.; Johansson, E. M. J.; Rensmo, H. Electronic 472 Structure of $\mathrm{TiO}_{2} / \mathrm{CH}_{3} \mathrm{NH}_{3} \mathrm{PbI}_{3}$ Perovskite Solar Cell Interfaces. J. 473 Phys. Chem. Lett. 2014, 5, 648-653.

474 (41) Varga, A.; Endrődi, B.; Hornok, V.; Visy, C.; Janáky, C. 475 Controlled Photocatalytic Deposition of CdS Nanoparticles on 476 Poly(3-hexylthiophene) Nanofibers: A Versatile Approach to Obtain 477 Organic/Inorganic Hybrid Semiconductor Assemblies. J. Phys. Chem. 478 C 2015, 119, 28020-28027.

479 (42) Endrodi, B.; Mellar, J.; Gingl, Z.; Visy, C.; Janaky, C. Reasons 480 Behind the Improved Thermoelectric Properties of Poly(3-hexylth481 iophene) Nanofiber Networks. RSC Adv. 2014, 4, 55328-55333.

482 (43) Duong, D. T.; Wang, C.; Antono, E.; Toney, M. F.; Salleo, A. 483 The Chemical and Structural Origin of Efficient p-Type Doping in 484 P3HT. Org. Electron. 2013, 14, 1330-1336.

485 (44) Weidman, M. C.; Seitz, M.; Stranks, S. D.; Tisdale, W. A. Highly 486 Tunable Colloidal Perovskite Nanoplatelets through Variable Cation, 487 Metal, and Halide Composition. ACS Nano 2016, 10, 7830-7839.

488 (45) Niklas, J.; Poluektov, O. G. Charge Transfer Processes in OPV 489 Materials as Revealed by EPR Spectroscopy. Adv. Energy Mater. 2017, $4907,1602226$.

491 (46) Aguirre, A.; Gast, P.; Orlinskii, S.; Akimoto, I.; Groenen, E. J. J.; 492 El Mkami, H.; Goovaerts, E.; Van Doorslaer, S. Multifrequency EPR 493 Analysis of the Positive Polaron in I2-Doped Poly(3-hexylthiophene) 494 and in Poly[2-methoxy-5-(3,7-dimethyloctyloxy)]-1,4-phenyleneviny495 lene. Phys. Chem. Chem. Phys. 2008, 10, 7129-7138.

496 (47) Camaioni, N.; Tinti, F.; Franco, L.; Fabris, M.; Toffoletti, A.; 497 Ruzzi, M.; Montanari, L.; Bonoldi, L.; Pellegrino, A.; Calabrese, A.; 498 et al. Effect of Residual Catalyst on Solar Cells Made of a Fluorene499 Thiophene-Benzothiadiazole Copolymer as Electron-Donor: A 500 Combined Electrical and Photophysical Study. Org. Electron. 2012, 501 13, 550-559.

502 (48) Pan, J.; Quan, L. N.; Zhao, Y.; Peng, W.; Murali, B.; Sarmah, S. 503 P.; Yuan, M.; Sinatra, L.; Alyami, N. M.; Liu, J.; et al. Highly Efficient 504 Perovskite-Quantum-Dot Light-Emitting Diodes by Surface Engineer505 ing. Adv. Mater. 2016, 28, 8718-8725.

506 (49) Pan, J.; Sarmah, S. P.; Murali, B.; Dursun, I.; Peng, W.; Parida, 507 M. R.; Liu, J.; Sinatra, L.; Alyami, N.; Zhao, C.; et al. Air-Stable 508 Surface-Passivated Perovskite Quantum Dots for Ultra-Robust, Single509 and Two-Photon-Induced Amplified Spontaneous Emission. J. Phys. 510 Chem. Lett. 2015, 6, 5027-5033.

511 (50) Krinichnyi, V. I. Multi Frequency EPR Spectroscopy of Conjugated 512 Polymers and Their Nanocomposites; CRC Press LLC: Boca Raton, FL, 5132016.

514 (51) Spano, F. C. The Spectral Signatures of Frenkel Polarons in H515 and J-Aggregates. Acc. Chem. Res. 2010, 43, 429-439.

516 (52) Clark, J.; Silva, C.; Friend, R. H.; Spano, F. C. Role of 517 Intermolecular Coupling in the Photophysics of Disordered Organic 518 Semiconductors: Aggregate Emission in Regioregular Polythiophene. 519 Phys. Rev. Lett. 2007, 98, 206406. 